\title{
How to Evaluate Creativity in Infancy. A New Method for 3/4 Year-Old Children ${ }^{+}$
}

\author{
Paola Molina ${ }^{1, *}$ and Benedetta Frezzotti ${ }^{2}$ \\ 1 Dipartimento di Psicologia, Università Degli Studi di Torino, 10124 Torino, Italy \\ 2 Studio Platypus, IED, 20100 Milano, Italy; benedetta.frezzotti@studioplatypus.it \\ * Correspondence: paola.molina@unito.it ; Tel.: +39-349-107-2817 \\ + Presented at the International and Interdisciplinary Conference IMMAGINI? Image and Imagination \\ between Representation, Communication, Education and Psychology, Brixen, Italy, 27-28 November 2017.
}

Published: 24 November 2017

\begin{abstract}
Our paper presents the preliminary results of a study aimed at building a new creativity assessment tool, suitable also for younger children (3/4 years old), who do not master enough language and design to respond to the classical tests used to evaluate this competence. The first results are encouraging, both for the collaboration and interest of children for the test, and for correlations with a classical evaluation tool used too (a task of the Torrance' TTCT). The discussion of results considers in particular the relationship between cognitive skills (representation) and individual response strategies.
\end{abstract}

Keywords: infancy; creativity; test; Torrance test; representation; image

\section{Introduction}

We define creativity in a Vygotskian perspective [1], as an re-elaboration, in a new and creative way, of elements necessarily present in the child's experience: the more this experience is rich, the more are the possibilities to creatively re-elaborate this experience.

A number of different tools to evaluate creativity in children are available (for a review, see [2], some adapted from adults instruments, others conceived expressly for children. Almost all these instruments utilize two principal tools: language or drawing, or a combination of both (verbal and figural forms).

The figural tasks requires the completion of non-significant drawing, to produce as many as possible different images, as the Torrance Tests of Creative Thinking-Visual form (TTCT) [3]. Another way to assess creativity (verbal tasks) consists in listing as many possible uses for a common house hold item (such as s brick, a paperclip, a newspaper, etc.), as in the Torrance Tests of Creative ThinkingVerbal form (TTCT) [3], or the Guilford's Alternative Uses Task [4].

Younger children however are not able enough in narrative discourse and drawing to allowing a reliable evaluation using such instruments. Therefore, we decided to experiment a new tool, allowing children to respond in a non-verbal manner, and without using the drawing.

Moreover, from a theoretical point of view, it is very difficult to define what is creative for very young children, because it is well know that some expression apparently creative for adults, seems rather derive from a lack of categorisation in thinking abilities of children. Tools allowing investigation of these aspects are therefore interesting in different field of research.

\section{Materials and Methods}

We collected the data during an ampler research on the 3-4 year-old children, observing children attending the first year of nursery schools in a small town of Piedmont (Italy). 13 nursery schools were involved, 9 public and 4 private. 
Table 1 reports participants in the study. We observed 69 children ( 32 girls); 19 children (11 girls) performed both tasks, 63 (28 girls) performed the Toy Improvement Task (TTCT-4 Task), and 27 (15 girls) performed the new task (IC: Image Completion). The children' mean age was 47 months (range 40-53 months).

Table 1. Participants.

\begin{tabular}{cccc}
\hline Tool & Boys (No Resp.) & Girls (No Resp.) & Total (No Resp.) \\
\hline Toy Improvement Task & $31(3)$ & $27(2)$ & $58(5)$ \\
Image completion & $12(0)$ & $15(0)$ & $27(0)$ \\
Both & $7(1)$ & $11(0)$ & $18(1)$ \\
\hline
\end{tabular}

We obtained the agreement from the heads and the teachers of the schools, and individually from both parents of each child. We observed the children using different tools, in a quiet room of the school. Moreover, parents filled questionnaires about family data and children' temperament. The administration order of the tests was not randomized, but depending of the school organization and of children' availability. We collected the data between May and June 2014.

We utilised two different tools.

\subsection{The Torrance Tests of Creative Thinking - Verbal Form (TTCT) - Task 4 (Improve a Toy)}

The task No. 4 (Improve a toy) was the only accessible for children in our sample. Despite this, 10 children refused to participate and 5 other children did not respond, although the test administrators were trained to solicit the answer in several ways.

The experimenter asked to the child "Try to improve this stuffed toy elephant so that it will be more fun to play with" (see Figure 1).

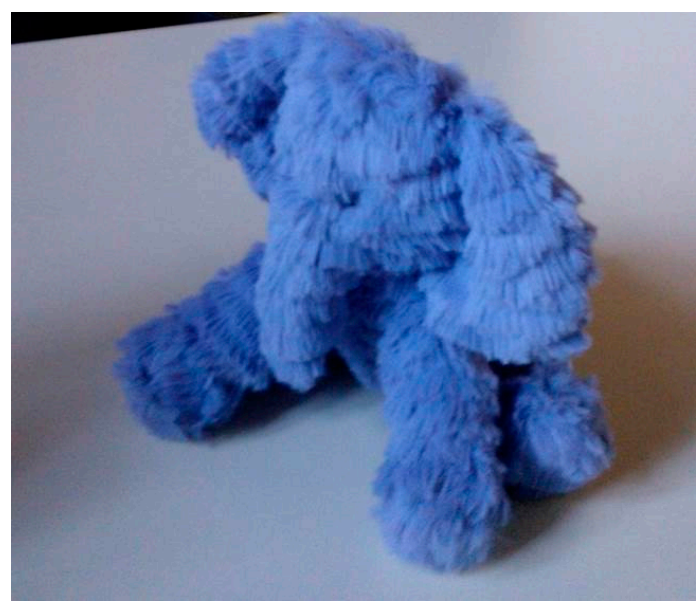

Figure 1. The stuffed toy elephant.

Children' responses were coded in terms of:

- $\quad$ FLUIDITY: Is the ability to produce a large number possible modifications

- $\quad$ FLEXIBILITY: Is the ability to produce different possible modifications (22 categories)

- ORIGINALITY: Is the ability to produce uncommon or unique responses that require creative strength.

We did not consider the optional criterion of ELABORATION (the ability to develop, embroider, embellish, carry out, i.e., elaborate ideas), because it was not adequate to the children' age.

All the tasks were audio-recorded, transcribed, and coded. 


\subsection{The Image Completion Task (ICT)}

In addition, we have expressly designed a new task of completing figures, the Image Completion Task (ICT). (See Figure 2).

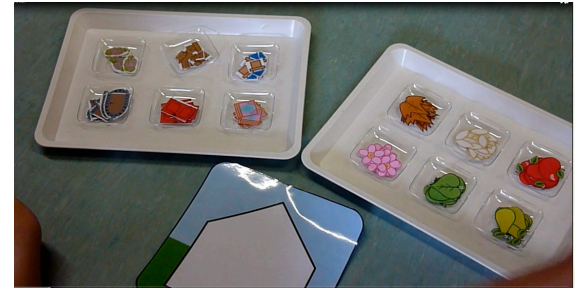

(a)

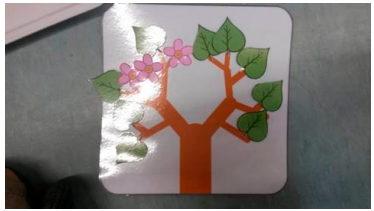

(b)

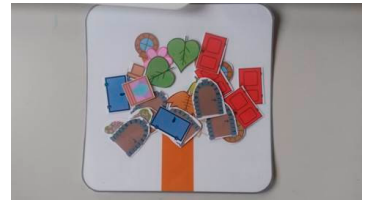

(c)

Figure 2. (a) The ICT materials; (b) Congruent; (c) Mixed.

We asked to the children to complete and make "as beautiful as possible" an image (a silhouette of a face, a house, a tree or a flower), using two different set of catted-out pieces (See Figure 2a), the first one congruent with the image, the second not congruent: for instance, we offered the silhouette of a house, and the pieces to complete the house (doors, windows, funnel, etc.) or to complete the image of a tree (flowers, fruits, leafs, etc.). We coded the images created by the children with different scores: the total NUMBER of pieces utilized, the number of CONGRUENT (See Figure $2 b$ ) and INCONGRUENT pieces. Moreover, we coded the use of MIXED pieces (congruent and incongruent, see Figure 2c) or not. Each child produced two different images, and we videotaped the task and coded the videos and the produced images. Moreover, we built a total score as the average of the two image scores.

Our research aims to answer two main questions:

1. The validity of the tasks:

a. Did the children participate?

b. There is a correlation among the scores, corresponding to what is expected by the instrument design?

2. There is a correlation among the two task allowing to validate the new tool (i.e., consistent with what we expected on the base of tool design)?

Moreover, we controlled possible difference by gender and presence of siblings.

\section{Results}

\subsection{Validity of the Tasks}

\subsubsection{Toy Improvement Task (TIT)}

First, we have verified the adequacy of the Toy Improvement Task (TIT) for children in our sample. As we have seen before, 10 children did not want to participate and 5 of those who accepted did not respond, just playing with the elephant.

58 children responded, and the three scores of Fluidity, Flexibility and Originality correlated with each other as expected, based on the instrument design (See Table 2).

Table 2. Correlation among the scores of the Toy Improvement Task $(\mathrm{N}=58)$.

\begin{tabular}{ccc}
\hline & Fluidity & Flexibility \\
\hline Fluidity & - & \\
Flexibility & $0.77^{1}$ & - \\
Originality & $0.70^{1}$ & $0.58^{1}$ \\
\hline
\end{tabular}

${ }_{1}^{1}$ Pearson correlation, two-tails, $p<0.001$. 
We did not found any difference by gender or presence of siblings: the Fluidity, Flexibility and Originality mean scores are reported in Table 3.

Table 3. Mean Scores of the Toy Improvement Task.

\begin{tabular}{cccccc}
\hline & N. & Minimum & Maximum & Mean & SD \\
\hline Fluidity & 58 & 1.00 & 18.00 & 6.47 & 4.374 \\
Flexibility & 58 & 1.00 & 7.00 & 2.72 & 1.715 \\
Originality & 58 & 0.00 & 16.00 & 3.66 & 3.967 \\
\hline
\end{tabular}

\subsubsection{The Image Completion Task (ICT)}

As for the new trial, the Image Completion Task (ICT), all the children (27) agreed to participate and completed two different images (one child asked to make a third), dedicating to the task a long enough time.

Comparing the scores of the first and second images (see Table 4), we observed that the use of congruent pieces was not related, while there was a substantial correlation between the number of pieces used and the tendency to use pieces of both categories (mixed). The correlation between the use of non-congruent pieces in the production of the two images was relevant (0.35), although not significant. There were no differences between the mean scores of the two images.

In addition, we found a correlation profile consistent with what we expected (see Table 5): both in the first and in the second image, the number of pieces was correlated to all the scores, the use of congruent pieces was not related to the two other scores, while non-congruent and mixed scores were related to each other.

We did not found any difference by gender or presence of siblings: the Number, Congruent, Incongruent and Mixed mean scores are reported in Table 4.

Table 4. Mean Scores in the Image Completion Tasks (ICT) and correlation between Image 1 and 2.

\begin{tabular}{ccccccc}
\hline Score & N. & Image 1 & Image 2 & $p^{1}$ & $r$ & $p^{2}$ \\
\hline Number & 27 & $11.74(8.08)$ & $10.15(6.11)$ & NS & 0.573 & 0.002 \\
Congruent & 27 & $6.74(5.85)$ & $5.76(6.37)$ & NS & -0.055 & 0.785 \\
$\begin{array}{c}\text { Incongruent } \\
\text { Mixed }\end{array}$ & 27 & $5.00(6.35)$ & $4.19(3.49)$ & NS & 0.353 & 0.071 \\
\hline
\end{tabular}

${ }^{1}$ Paired samples $t$-test, two-tails; ${ }^{2}$ Pearson correlation, two-tails.

Table 5. Correlation among the scores of the Image Completion Task (ICT), image 1 and $2(\mathrm{~N}=27)$.

\begin{tabular}{ccccccc}
\hline & \multicolumn{3}{c}{ Image 1 } & \multicolumn{3}{c}{ Image 2 } \\
\hline Score & Number & Congruent & Incongruent & Number & Congruent & Incongruent \\
\hline Number & - & & & - & & \\
Congruent & $0.63^{3}$ & - & - & $0.84^{3}$ & - & - \\
Incongruent & $0.70^{3}$ & -0.12 & 0.21 & -0.35 & 0.10 & $0.50^{2}$ \\
Mixed & $0.42^{2}$ & -0.20 & $0.72^{3}$ & $0.39^{1}$ & 0.10 \\
\hline
\end{tabular}

Pearson correlation, two-tails, ${ }^{1}=p<0.05 ;^{2}=p<0.01 ;^{3}=p<0.001$.

\subsection{The Relationship among Tasks}

18 children completed both tasks: we correlated the scores of TIT with the scores constructed from the two images in the ICT (See Table 6). Correlations are not significant due to the limitation of our sample, but the correlation profile is consistent with the expected profile: the number of pieces used as well as the number of congruent pieces do not correlate with the scores of TIT, while the correlation between the number of incongruent pieces with scores of Fluidity and Flexibility of TIT are substantive, and the use of mixed pieces correlates with all three scores of TIT. 
Table 6. Pearson correlation scores between instruments $(\mathrm{N}=18)$.

\begin{tabular}{cccc}
\hline & \multicolumn{3}{c}{ Scores TIT } \\
\hline Scores ICT & Fluidity & Flexibility & Originality \\
\hline Number & 0.18 & 0.18 & -0.04 \\
Congruent & -0.03 & -0.03 & -0.10 \\
Incongruent & 0.32 & 0.32 & 0.04 \\
Mixed & 0.32 & 0.32 & 0.31 \\
\hline
\end{tabular}

\section{Discussion}

Our goal was to develop a new test of creativity, suitable for very young children. The underlying idea was to be able to differentiate children in relation to their ability not to confine themselves to the use of canonical materials in building the image, but to use different materials flexibly.

The work presented here is a preliminary work, of an exploratory nature, on a still small sample. Nevertheless, it shows some interesting aspects: children seem to use different strategies, which can be linked to those evaluated with other tools such as the TIT.

However, we cannot overlook the problematic aspects.

First of all, it is difficult to use an effective request: classical request of tests like the TTCT is very rigid, and it seems unclear for children of this age. On the other hand, a friendlier expression is likely to suggest to the child possible strategies, not automatically present [5].

Moreover, the validation of the instrument will require a careful qualitative analysis of the strategies used by children to build a beautiful image, strategies that have proved to be very different (use all elements (Figure 3a), aesthetic criterion (Figure 3b), do not leave white space (Figure 3c), etc.: see Figure 3), which does not always correspond to adult aesthetic criteria.

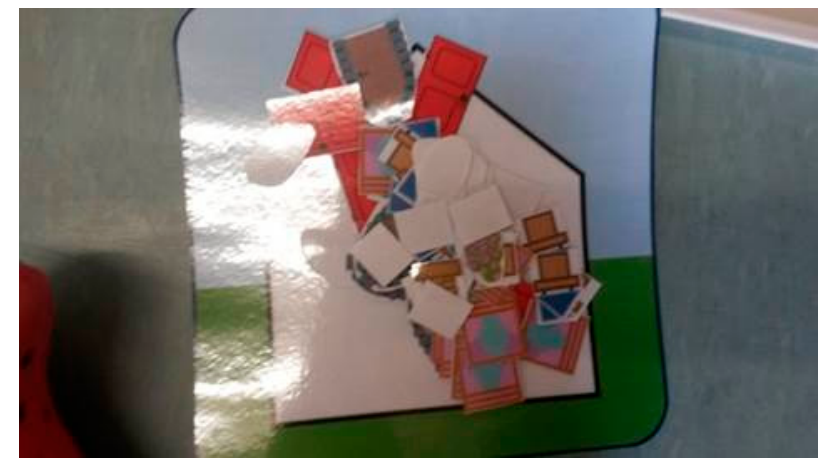

(a)

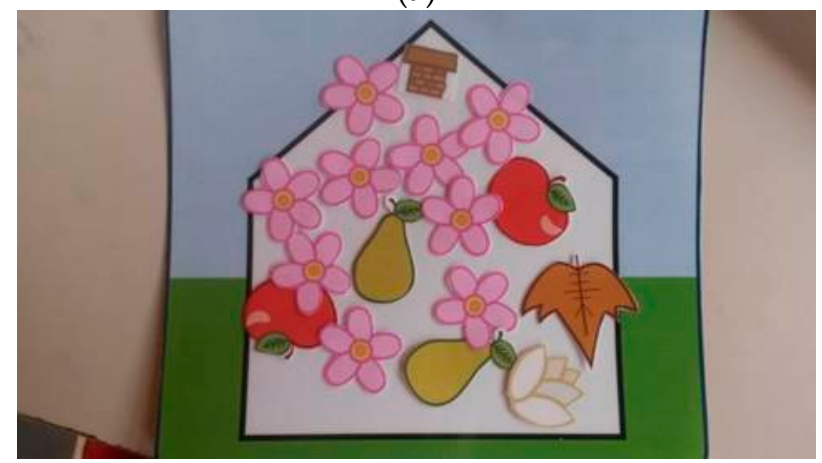

(b) 


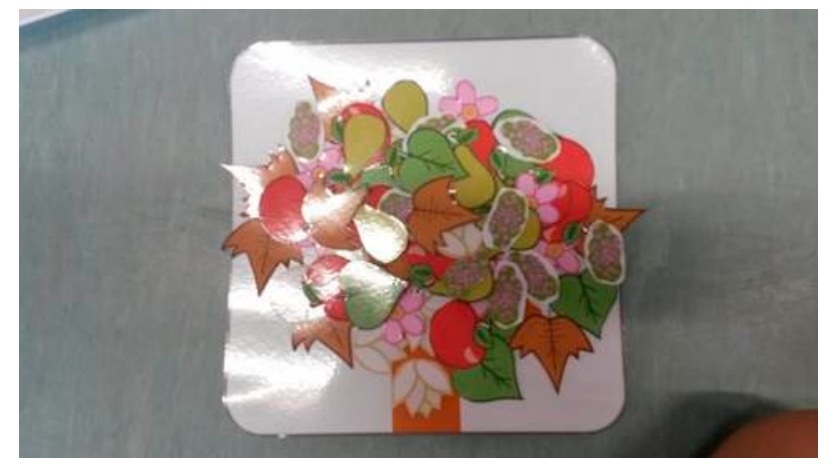

(c)

Figure 3. Beautiful is (a) Use all elements; (b) Aesthetic criterion ("These flowers are beautiful"); (c) Do not leave white space.

It is also necessary to better understand whether the difficulties that younger children have in drawing, also appear in the completion of images, thus linked not to motor skills but to a representation problem. As can be seen in Figure 4, although the drawing difficulties are overcome by the use of pre-cut forms, the same cannot be said of the difficulties in using symbolic representation: composition 4c may be beautiful, that is, it can be done by the child with aesthetic criteria [6], but does not represent a face; the mere use of the proposed quantitative criteria risks neglecting a fundamental aspect in determining the child's response.

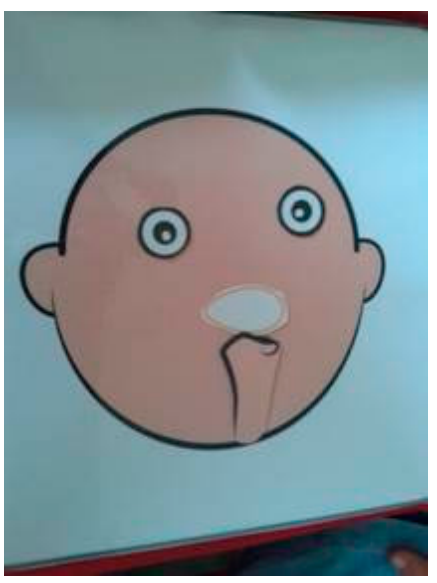

(a)

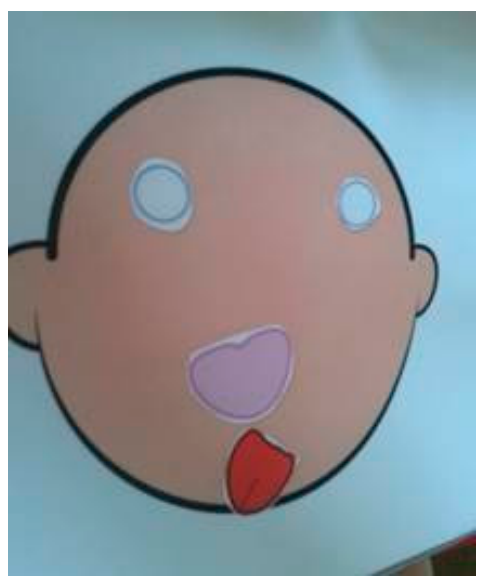

(b)

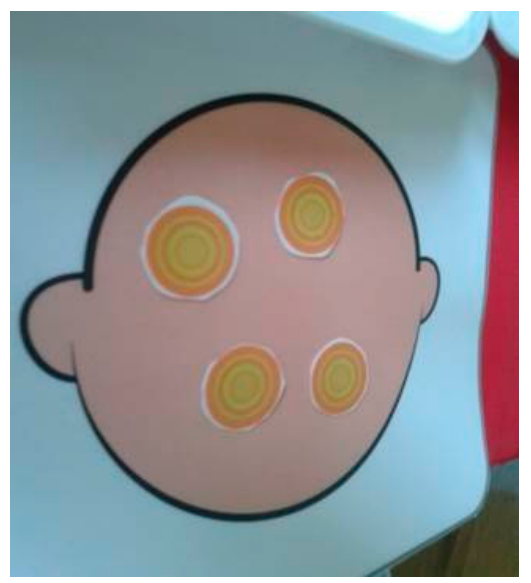

(c)

Figure 4. Children' different strategies to complete the face. (a) Almost canonical; (b) Not canonical; (c) Still a face?

Even for TIT testing, which we used to validate the new tool, it seems necessary to detect some problematic aspects.

First, the available standardization is inadequate, because it is distant over time and because it is not suitable to differentiate children in different age groups. In addition, although the manipulation of the concrete object (the stuffed elephant) proved to be an age-appropriate strategy, children are not always able to understand the request, as evidenced by the relatively high number of children who did not respond, but they just played with the elephant.

\section{Conclusions}

Both tools we used show results consistent with what theoretically hypothesized. As far as the comparison between the two tasks is concerned, although the results are not significant (even because the numbers are very low), the correlation profile between the two tools can be considered as a first confirmation of the usefulness of the new procedure for early estimation of creativity, in relation to the linguistic and cognitive limited skills of children. The new tool that we present here, although 
still needs adequate standardization work, is therefore an interesting tool for evaluating creativity in preschool age, where similar instruments are still lacking.

In the future, in addition to expanding the sample and controlling relationships with other skills implicated in the performance of children, especially the cognitive and linguistic ones, we plan to use the tool even with adults and older children.

Acknowledgments: The Fondazione Ferrero (Alba) financed the research in the years 2012-2015. We are grateful to the children who participated in the study, to their families and their teachers. We thank the bachelor and master students who gave their contribution in data collection.

Author Contributions: Paola Molina conceived and designed the research, collected and analysed the data, and wrote the paper. Benedetta Frezzotti conceived the tool, realized the prototype and revised the manuscript.

Conflicts of Interest: The authors declare no conflict of interest.

\section{References}

1. Vygotskij, L.S. Voobraženie i Tvorčstvo v Škol'nom Vozraste; Gosudarstvennoe Izdatel'stvo: MoskvaLeningrad, URSS, 1930. (Italian Version: Immaginazione e Creatività nell'età Infantile; Editori Riuniti: Roma, Italy, 1972).

2. Santoro, M. Creatività Nell'età Evolutiva: Teorie e Strumenti di Valutazione. Master Degree Dissertation, Università Degli Studi di Torino, Torino, Italy, 2015

3. Torrance, E.P. Torrance Tests of Creative Thinking, 3rd ed.; Scholastic Tesing Service Inc.: Bensenville, IL, USA, 1987. (Italian Version: Sprini G. \& Tommasiello S., Test di Pensiero Creativo. Manuale e Guida alla Valutazione; O.S. Organizzazioni Speciali: Firenze, Italy, 1989).

4. Guilford, J.P.; Christensen, P.R.; Merrifield, P.R.; Wilson, R.C. Alternate Uses: Manual of Instructions and Interpretations; Sheridan Psychological Services: Orange, CA, USA, 1978.

5. Piaget, J. La Représentation du Monde chez L'Enfant; Presses Universitaires de France: Paris, France, 1926.

6. Kellog, R. Analysing Children's Art; Mayfield: Palo Alto, CA, USA, 1970.

(C) 2017 by the authors. Licensee MDPI, Basel, Switzerland. This article is an open access article distributed under the terms and conditions of the Creative Commons Attribution (CC BY) license (http://creativecommons.org/licenses/by/4.0/). 